\title{
A Framework for Local Mechanical Characterization of Atherosclerotic Plaques: Combination of Ultrasound Displacement Imaging and Inverse Finite Element Analysis
}

\author{
Ali C. Akyildiz, ${ }^{1,4}$ Hendrik H. G. Hansen, ${ }^{2}$ Harm A. Nieumstadt, ${ }^{1}$ Lambert Speelman, ${ }^{1}$ \\ Chris L. De Korte, ${ }^{2}$ Antonius F. W. van der Steen, ${ }^{1,3}$ and Frank J. H. Gijsen ${ }^{1}$ \\ ${ }^{1}$ Biomechanics Lab, Department of Biomedical Engineering, Thoraxcenter, Erasmus Medical Center, PO Box 2040,3000 \\ CA Rotterdam, The Netherlands; ${ }^{2}$ Medical UltraSound Imaging Center (MUSIC), Department of Radiology and Nuclear \\ Medicine, Radboud University Medical Center, Nijmegen, The Netherlands; ${ }^{3}$ Department of Applied Sciences, Delft University \\ of Technology, Delft, The Netherlands; and ${ }^{4}$ Department of Mechanical, Aerospace and Nuclear Engineering, Rensselaer \\ Polytechnic Institute, Troy, USA
}

(Received 11 January 2015; accepted 24 July 2015; published online 23 September 2015)

Associate Editor Andreas Anayiotos oversaw the review of this article.

\begin{abstract}
Biomechanical models have the potential to predict plaque rupture. For reliable models, correct material properties of plaque components are a prerequisite. This study presents a new technique, where high resolution ultrasound displacement imaging and inverse finite element (FE) modeling is combined, to estimate material properties of plaque components. Iliac arteries with plaques were excised from 6 atherosclerotic pigs and subjected to an inflation test with pressures ranging from 10 to $120 \mathrm{mmHg}$. The arteries were imaged with high frequency $40 \mathrm{MHz}$ ultrasound. Deformation maps of the plaques were reconstructed by cross correlation of the ultrasound radiofrequency data. Subsequently, the arteries were perfusion fixed for histology and structural components were identified. The histological data were registered to the ultrasound data to construct FE model of the plaques. Material properties of the arterial wall and the intima of the atherosclerotic plaques were estimated using a grid search method. The computed displacement fields showed good agreement with the measured displacement fields, implying that the FE models were able to capture local inhomogeneities within the plaque. On average, nonlinear stiffening of both the wall and the intima was observed, and the wall of the atheroslcerotic porcine iliac arteries was markedly stiffer than the intima $(877 \pm 459$ vs. $100 \pm 68 \mathrm{kPa}$ at $100 \mathrm{mmHg})$. The large spread in the data further illustrates the wide variation of the material properties. We demonstrated the feasibility of a mixed experimental-numerical framework to determine the material properties of arterial wall and intima of atheroscle-
\end{abstract}

Address correspondence to Frank J. H. Gijsen, Biomechanics Lab, Department of Biomedical Engineering, Thoraxcenter, Erasmus Medical Center, PO Box 2040, 3000 CA Rotterdam, The Netherlands. Electronic mails: acakyildiz@gmail.com and f.gijsen@ erasmusmc.nl rotic plaques from intact arteries, and concluded that, due to the observed variation, plaque specific properties are required for accurate stress simulations.

Keywords-Material properties, Atherosclerotic plaque, Inverse method, Ultrasound.

\section{INTRODUCTION}

Atherosclerotic plaques are characterized by local thickening of the arterial vessel wall, mainly caused by lipid and inflammatory cell infiltration, smooth muscle cell migration and proliferation and extracellular matrix buildup. ${ }^{11}$ Some atherosclerotic plaques have a higher chance of causing clinical events and are therefore called vulnerable plaques. Vulnerable plaques are morphologically characterized by a large lipid core and a relatively thin fibrous cap, separating the lipid core from the lumen. ${ }^{39}$ Rupture of the cap exposes the content of the lipid core to the blood stream and leads to intraluminal thrombosis. Thrombosis triggered by plaque rupture is the predominant cause of myocardial infarction and stroke. ${ }^{44}$

Today means of accurately predicting the rupture risk of a plaque are still lacking. Biomechanical studies showed that high stress regions in atherosclerotic plaques correspond to rupture locations. ${ }^{9,37}$ The rationale behind this is that rupture occurs when the stress at a certain location inside the cap exceeds the local cap strength. Biomechanical models that are used to compare computed cap stresses with cap strength have 
the potential to improve plaque rupture risk assessment. ${ }^{18}$ For accurate stress calculations, biomechanical plaque models not only rely strongly on the plaque geometry, but also on the material properties of plaque components. ${ }^{2,35}$ However, experimental data for plaque properties are scarce and available data span a wide range. ${ }^{1,45}$

One of the underlying reasons for the wide spread in the experimental data is that commonly applied testing methods generally involve loss of plaque integrity. By testing atherosclerotic plaques with in vivo deformation measurements or ex vivo inflation tests on the other hand, the structural integrity of the tissue is not compromised. Ex vivo inflation tests have been widely used to estimate material properties of healthy vessels. ${ }^{19,43}$ Generally, the deformation of the outer border of the vessel wall was measured. The vessel was modeled as a thin walled cylinder and the material properties were obtained by solving the problem analytically. ${ }^{14,22,36}$ Due to the complex morphology of atherosclerotic plaques, determination of the material properties cannot be done with analytical solutions and requires more advanced approaches such as inverse FE analysis.

With inverse FE analysis, material properties can be estimated by adjusting material parameters in the FE simulations iteratively and minimizing the difference between the computed and measured deformations. The feasibility of this methodology was demonstrated using synthetic, simulated inflation test results as an input. ${ }^{5,12,20,21,26}$ Other groups used the inverse FE based on global plaque deformation measurements, using either intravascular ultrasound $d^{8,15,32}$ or magnetic resonance imaging. ${ }^{28}$ The main drawback of using global deformation measurements is that the underlying FE model cannot incorporate multiple plaque components. Baldewsing et $a l^{3}$ applied intravascular ultrasound to obtain local deformation measurements, and they were able to demonstrate the feasibility of the method to generate elasticity maps of coronary plaques. The study focused on in vivo applications to determine the material properties for small incremental strain values, and the shape that individual plaque components could adopt to was restricted. Finally, Beatty et al. subjected aortic segments to inflation tests to determine material properties using optical techniques to measure the local displacement, ${ }^{4}$ thus limiting the future utilization of this technique in a clinic setting.

In this study the feasibility of a novel hybrid framework for mechanical characterization of atherosclerotic plaques based on ex vivo inflation tests is demonstrated. The method combines high resolution ultrasound deformation measurements and plaque morphology information with inverse $\mathrm{FE}$ analysis to compute local, nonlinear material properties of arterial wall and intima of atherosclerotic plaques. The feasibility of this framework was demonstrated by characterizing the properties of advanced atherosclerotic plaques from porcine iliac arteries.

\section{METHODS}

The methodology of the study consists of three main parts: ex vivo inflation tests, FE modeling, and estimation of material properties of plaque components (Fig. 1). The individual steps are explained below in details.

\section{Ex Vivo Inflation Tests}

Atherosclerotic iliac arteries $(n=6)$ were collected from diabetic pigs that were on a high cholesterol diet. Briefly, streptozotocin-induced diabetic pigs $(\sim 45 \mathrm{~kg})$ were fed supplemental ( $40 \%$ of dietary energy) saturated fat/cholesterol, unsaturated fat or starch for 10 weeks. The pigs showed substantial amount of atherosclerotic lesions in the arterial system. Details of this animal model can be found in previous work. ${ }^{24}$

Immediately after sacrificing the animals, iliac arteries were excised and snap-frozen in liquid nitrogen. Arteries were stored at $-80{ }^{\circ} \mathrm{C}$ until the day of inflation experiments. At the day of experimentation, the arteries were thawed to room temperature, cannulated and the side branches were closed by sutures. Based on the estimated in vivo length, the arteries were stretched $20 \%$ in longitudinal direction and preconditioned between 80 and $120 \mathrm{mmHg}$ for ten times. In the inflation experiments, intraluminal pressure was increased from 10 to $120 \mathrm{mmHg}$ in a quasi-static nature and measurements were acquired when the pressure and deformation stabilized. The tests were conducted in a custom build vessel perfusion system at room temperature.

Deformation of the atherosclerotic vessel wall during the inflation test was imaged with a Vevo ${ }^{\circledR}$ 2100 ultrasound system (FUJIFILM VisualSonics, Inc., Toronto, Canada) using a high frequency linear array transducer (MS550D with center frequency of $40 \mathrm{MHz}$ ). Before the inflation experiments, a $3 \mathrm{D}$ reconstruction of the segment was generated by stacking a series of $2 \mathrm{D}$ B-mode images using a $3 \mathrm{D}$ motor stage. The images were evaluated visually to identify a location with a substantial atherosclerotic plaque, at least $10 \mathrm{~mm}$ away from either cannula. The 


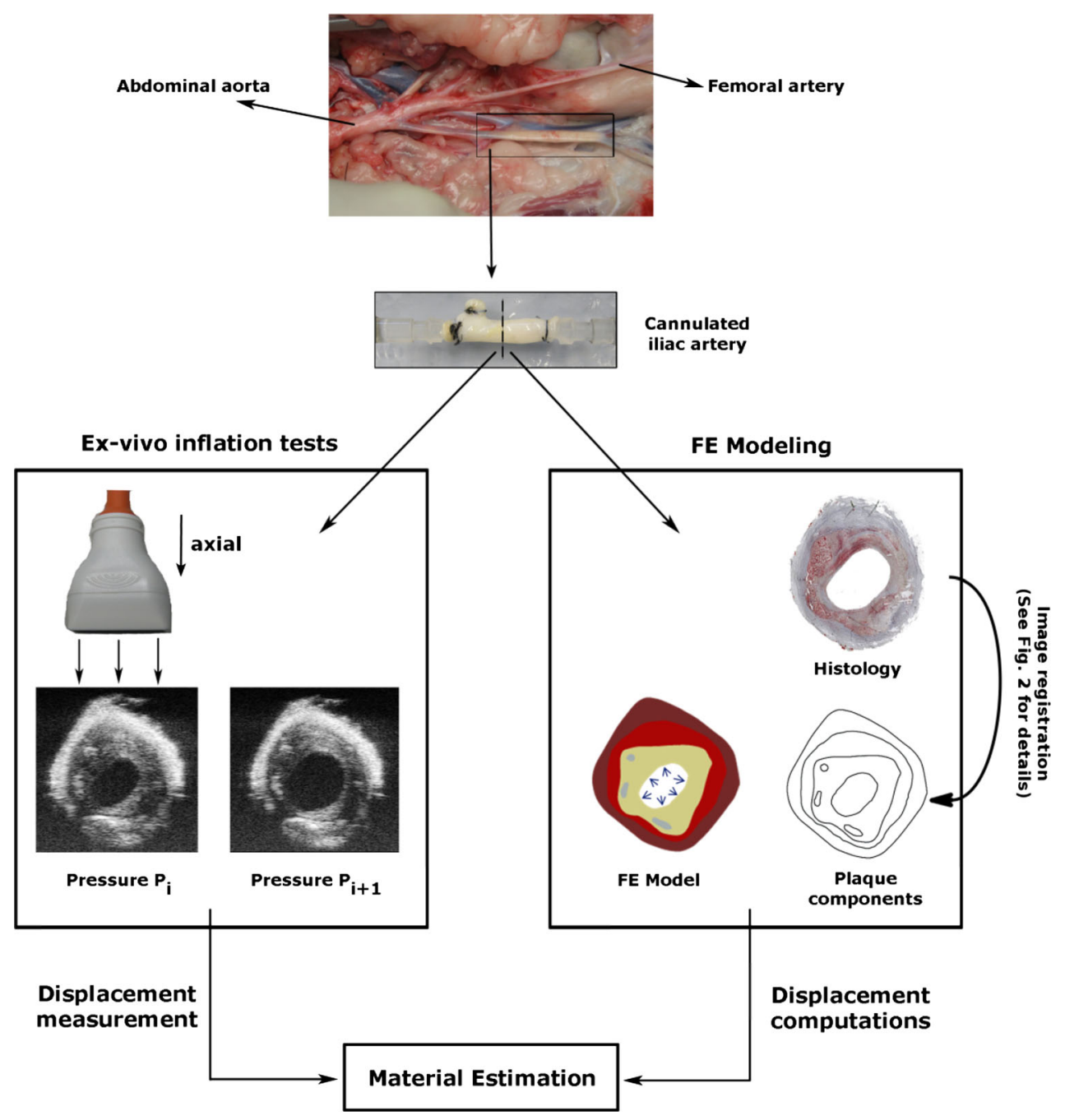

FIGURE 1. Methodology of the study consists of three main parts: ex vivo inflation tests, FE modeling, and estimation of material properties.

location was marked with a tissue marker pen on the outside of the vessel to find it accurately in a later phase for histology preparation. Transversal crosssectional B-mode ultrasound images and radiofrequency (RF) data were recorded at this location at each pressure step.

The RF data enabled us to calculate the axial (along the ultrasound lines) and lateral (in the direction perpendicular to ultrasound lines) displacements on the transversal plaque cross-section with high precision. Due to the availability of phase and amplitude information in axial direction, the displacement estimates in this direction are more accurate than in the lateral direction. The displacement estimation is performed in three iterations with a coarse-to-fine 2D cross-correlation method using RF data and is explained in detail elsewhere. ${ }^{29}$ The displacement estimation method was originally developed for clinical ultrasound scanners using center frequencies up to $10 \mathrm{MHz}$, and applied for high-frequency scanners for the first time. In the first iteration, template and search kernel sizes of $3850 \times 715 \mu \mathrm{m}^{2}($ axial $\times$ lateral $)$ and $7700 \times 935 \mu \mathrm{m}^{2}$ with a kernel overlap of $50 \%$ in the axial direction and a kernel overlap of $92 \%$ in the lateral direction were used to find a coarse displacement estimate. In the second iteration template and search windows sizes of $60 \times 715$ and $120 \times 935 \mu \mathrm{m}^{2}$ were used with a window overlap of $75 \%$ and $92 \%$ in the axial and lateral direction, respectively. In the last iteration local aligning of the data including parabolic interpolation of the cross-correlation peak was performed to obtain accurate subsample displacement estimates. The kernel sizes remained the same in iteration two and three. A median filter of $5 \times 5$ displacement pixels was applied after each iteration to decrease the amount of outlying displacement values. The final spatial resolution for the displacement estimates was $15 \mu \mathrm{m}$ in axial direction and $55 \mu \mathrm{m}$ in the lateral direction. 


\section{Finite Element Models}

Plaque morphology is crucial in FE models if mechanical characterization of individual plaque components is aimed for. Ultrasound imaging can provide this essential information to a certain degree, as sufficient contrast cannot be obtained to delineate all plaque components. Although lumen and outer vessel wall contours can be detected clearly and easily, media-intima interface and calcium regions could not be identified precisely. To overcome this problem, we used a hybrid approach in which histology images provide the detailed information of different plaque components (Fig. 2). To obtain the histological images, the arteries were fixed with formaldehyde for histology at $10 \mathrm{mmHg}$ and $20 \%$ longitudinal prestretch. One $5-\mu \mathrm{m}$ thick slice from each imaged plaque cross-section was used for Oil red O (ORO) staining, counter stained with hematoxylin. In ORO staining, lipids and fatty acids appear red. The counter-staining hematoxylin stains the nuclei and calcium blue. This staining enabled us to delineate the adventitia, media, intima, calcium, lumen and the outer border of the vessel wall.

The histological images were slightly different in shape and size than the B-mode images due to histology preparation. To ensure that the plaque morphology in the FE models accurately reflects the plaque morphology in the inflation tests, the histology images were registered to B-mode images. Contours of plaque components delineated on histology images were mapped on ultrasound images (Figs. 2c-2f) using the image registration software elastix. ${ }^{23}$ elastix is an open source software, based on the well-known Insight Segmentation Toolkit (ITK) and used for (medical) image registration. First, the lumen contour and the outer vessel wall contour were manually drawn both on the ultrasound image obtained at $10 \mathrm{mmHg}$ (Fig. 2c) and on the histology image (Fig. 2d). Subsequently, the contours on the images were mapped. This resulted in a transformation matrix from histology image to ultrasound image for the entire plaque crosssection. Finally, the contours of the plaque components drawn on the histology image (Fig. 2e) were transformed onto the ultrasound image (Fig. 2f) by using the transformation matrix obtained in the previous step. The transformed histology contours were used to create 2D FE models of the plaques with detailed morphology information (Fig. 2g).

Based on the histology images, FE models (ABAQUS, version 6.11) were created to simulate the plaque deformation during the inflation tests. The nonlinearity in the material behavior of plaque components was modeled with a nonlinear, incompressible Neo-Hookean model for all plaque components. Incompressible
Neo-Hookean material model is characterized by the strain energy density function $W$ defined as $W=C$ $\left(I_{1}-3\right)$, where $C$ is the shear modulus and $I_{1}$ is the first invariant of the left Cauchy-Green deformation tensor. Identical shear moduli were assigned to adventitia and media. This material complex is referred to as "wall" in the rest of the paper. Calcium was assumed to be very stiff $\left(C=10^{5} \mathrm{kPa}\right)$. To prevent rigid body motion in the FE simulations, a very soft and compressible solid buffer layer surrounding the plaque was created and zero-displacement boundary conditions were applied to its outer border. The models were pressurized intraluminally following the protocol of the inflation experiment and solved using previously developed numerical procedures. ${ }^{2,41}$ Linear triangular and quadrilateral plane strain elements were used in the FE models. Hybrid elements were preferred to avoid volumetric locking. The total number of elements in each model differed depending on the size and shape of the plaque cross-section and varied between $20 \times 10^{3}$ and $100 \times 10^{3}$. The FE models passed the checks on convergence and satisfaction of applied load and boundary conditions.

\section{Estimation of Material Properties}

A grid search method was employed for the material parameter estimation. The material parameters were altered within a prespecified range with a constant step size and all possible combinations of the parameters were simulated. In the estimation procedure, shear moduli of plaque wall and intima were varied between 1 and $400 \mathrm{kPa}$ in the FE models.

The measured displacements from central region of the plaques in the axial direction were used in the estimation procedure to find the optimum material properties. The axial direction of the ultrasound beam in this region corresponds to the radial direction in polar coordinates with the coordinate system center at the lumen center. Plaques were located in the test setup such that the thickest plaque section was in this region. A grid with $100 \mu \mathrm{m}$ element size $(\sim 6 \times$ the axial and $\sim 2 \times$ the lateral in-plane resolution of ultrasound data) was generated in this plaque region as illustrated in Fig. $2 \mathrm{~h}$. The total number of elements in the grids varied between 30 and 50 depending on the plaque thickness.

In each grid element, both the computed and measured displacements were averaged. To cover the material nonlinearity in the physiological pressure range in arteries of humans ( 80 to $120 \mathrm{mmHg}$ ), the displacements from 10 to $80 \mathrm{mmHg}$ (step 1), from 80 to $100 \mathrm{mmHg}$ (step 2), and from 100 to $120 \mathrm{mmHg}$ (step 3) were used in the analysis. An objective func- 


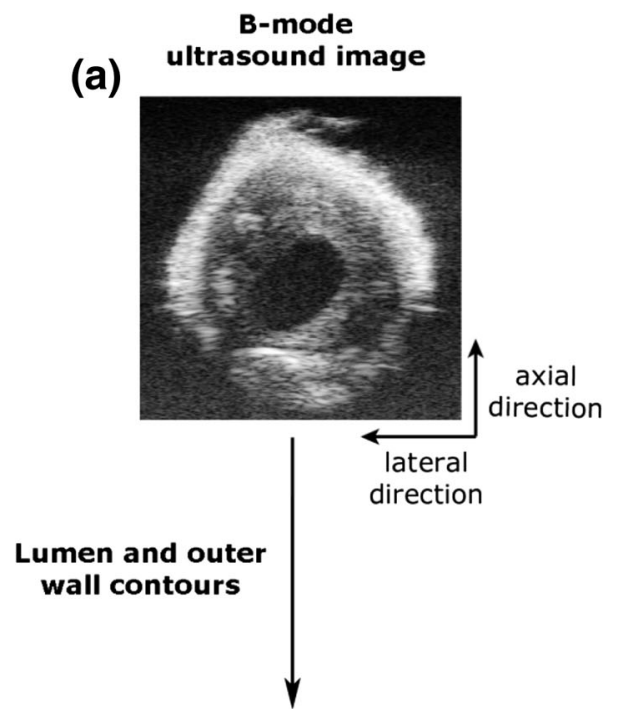

(c)

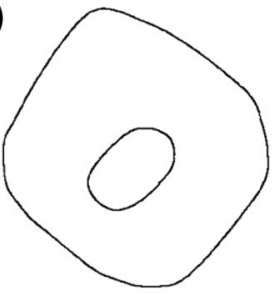

(b)

\section{Histology} image

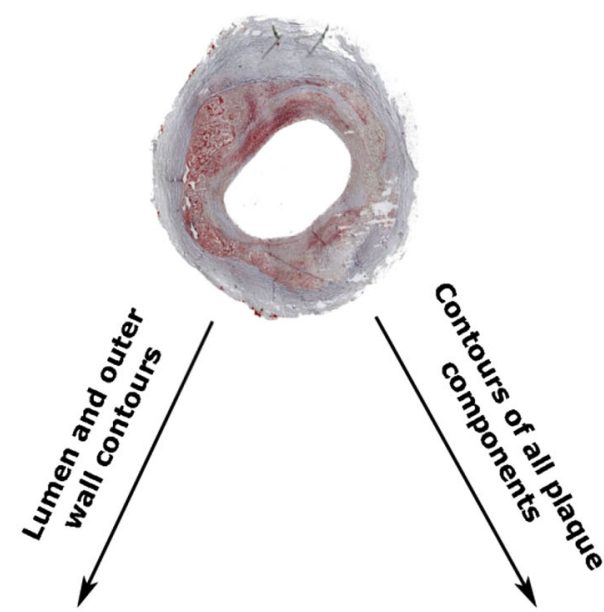

(d)

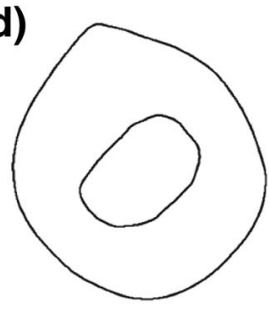

(e)

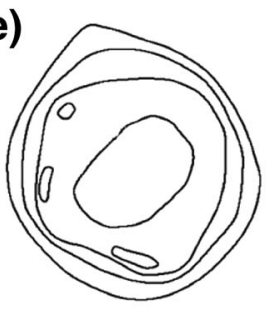

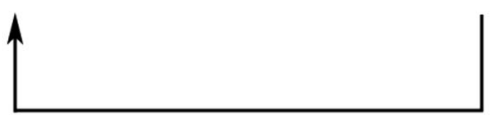

Transformation matrix

(from elastix)

Application of the transformation matrix (h)

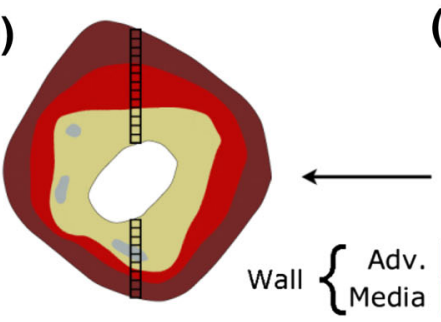

Grid for estimation procedure

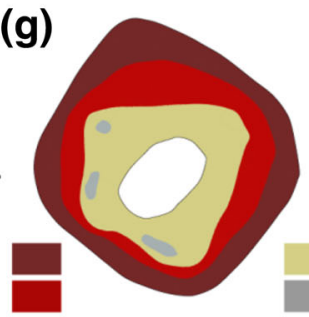

FE model

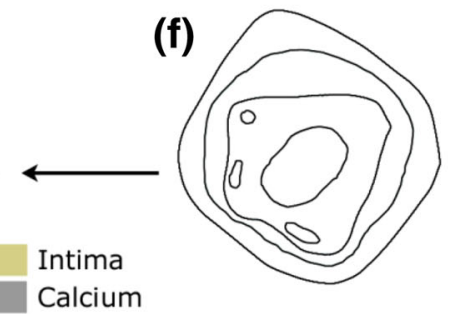

Contours transformed on ultrasound image

FIGURE 2. Illustration of how the geometry for FE models was obtained. Plaque component borders drawn on histology images were transformed onto the B-mode ultrasound image by the image registration software elastix. Axial (along the ultrasound lines) and lateral (in the direction perpendicular to ultrasound lines) directions are indicated next to the B-mode image.

tion, $F$, to be minimized in the estimation procedure for each pressure step was defined as

$$
F=\sum_{j=1}^{n}\left(u_{j}^{\text {comp }}-u_{j}^{\text {meas }}\right)^{2},
$$

similar as was done by Beattie et al. ${ }^{4}$ In the objection function, $F, u_{j}^{\text {comp }}$ and $u_{j}^{\text {meas }}$ are the average computed and measured displacement in each grid, respectively,
" $j$ " represents the grid element number and " $n$ " the total number of the grid elements. The FE simulation with the lowest value of the objective function, $F$, was considered as the best match to the experimental measurements and the shear moduli used in this FE model as the estimate of the intima and wall material properties. The estimation procedure was run for all 3 pressure steps separately to have shear modulus esti- 
Plaque 1
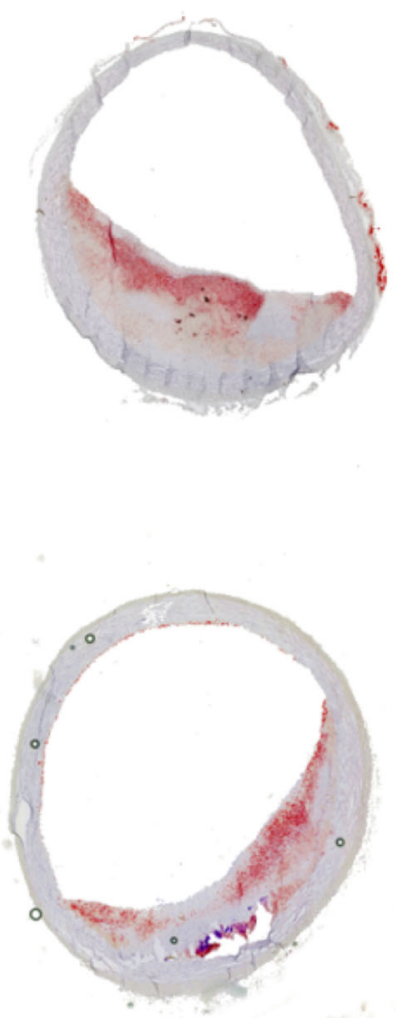

Plaque 4
Plaque 2
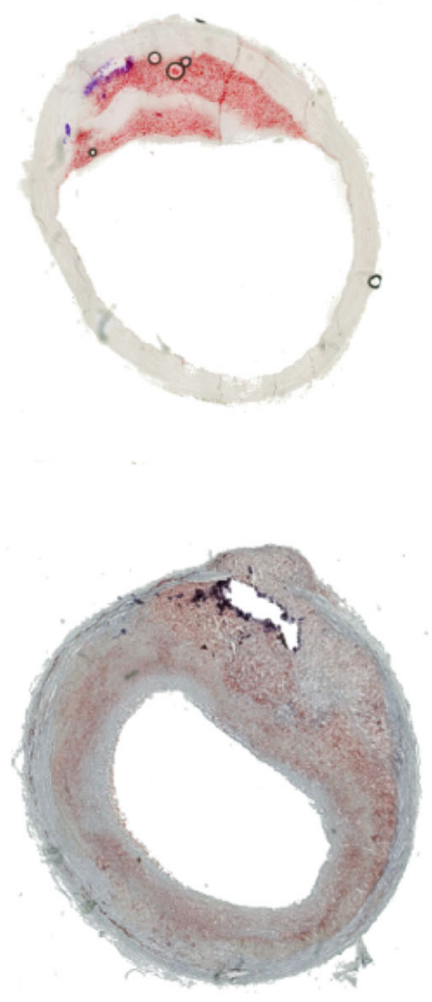

Plaque 5
Plaque 3
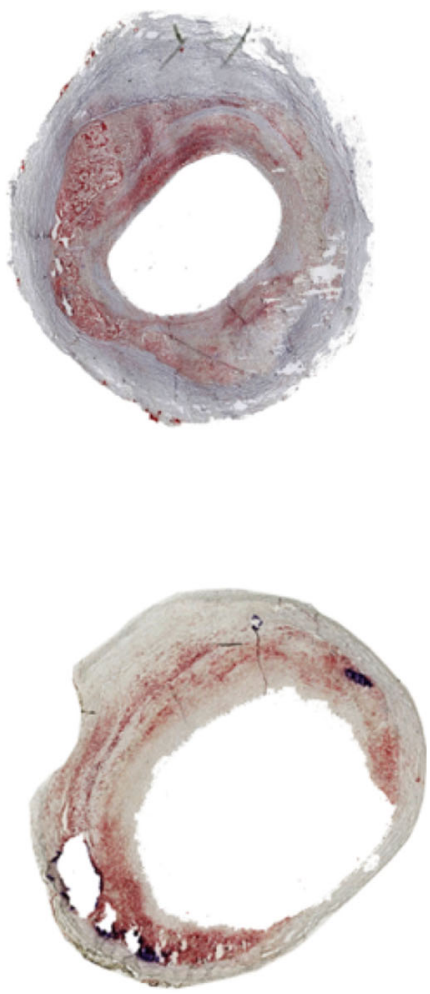

Plaque 6

FIGURE 3. Histology images of the atherosclerotic plaques from porcine iliac arteries. The holes with the blue outline in the images are where calcifications were present before they dissolved during the histology preparation.

mation for each pressure step. The corresponding Young's modulus values, $E$, of the estimated shear moduli were calculated with the formula, $E=6 * C$, and reported in the remainder of the paper for an easy comparison to the results reported in literature. The goodness of the parameter estimation for each plaque and each pressure step was evaluated by computing the relative difference between the measured and computed displacement:

$$
u_{\text {rel }}=\left[\left(\frac{\sqrt{F}}{n}\right)\left(u_{\text {mean }}^{\text {meas }}\right)^{-1}\right] \times 100 \%,
$$

where $u_{\text {mean }}^{\text {meas }}$ represents the average absolute displacement for each pressure step.

\section{RESULTS}

Of the six porcine iliac plaques, two plaques were concentric and four were eccentric. Figure 3 shows the histology images of the plaques. The atherosclerotic intima contained extracellular lipids and collagen fi- bers. No lipid pool or necrotic core was present in any of the plaques. All but plaque 1 contained calcium in varying sizes. The calcifications were localized usually near the intima-media interface.

Displacement measurements were successfully performed with the cross correlation technique applied to the RF data from high frequency ultrasound imaging. An example (Plaque 3) of the measured displacements from 10 to $80 \mathrm{mmHg}$ in the axial and lateral directions is shown in the upper panel of Fig. 4. Axial displacements showed a smooth profile. As expected, the lateral displacement measurements were less smooth. The corresponding computed displacements (of the simulation with the minimum objective function value) are shown in the lower panel of Fig. 4. The figure demonstrates that the principal displacements within the plaque were outwards in the radial direction, which is the expected deformation profile of a pressurized arterial segment.

Figure 5 represents the measured and computed axial displacements in the grid elements (lines $\mathrm{A}-\mathrm{B}$ and C-D) for all three pressure steps for plaque 3. For 


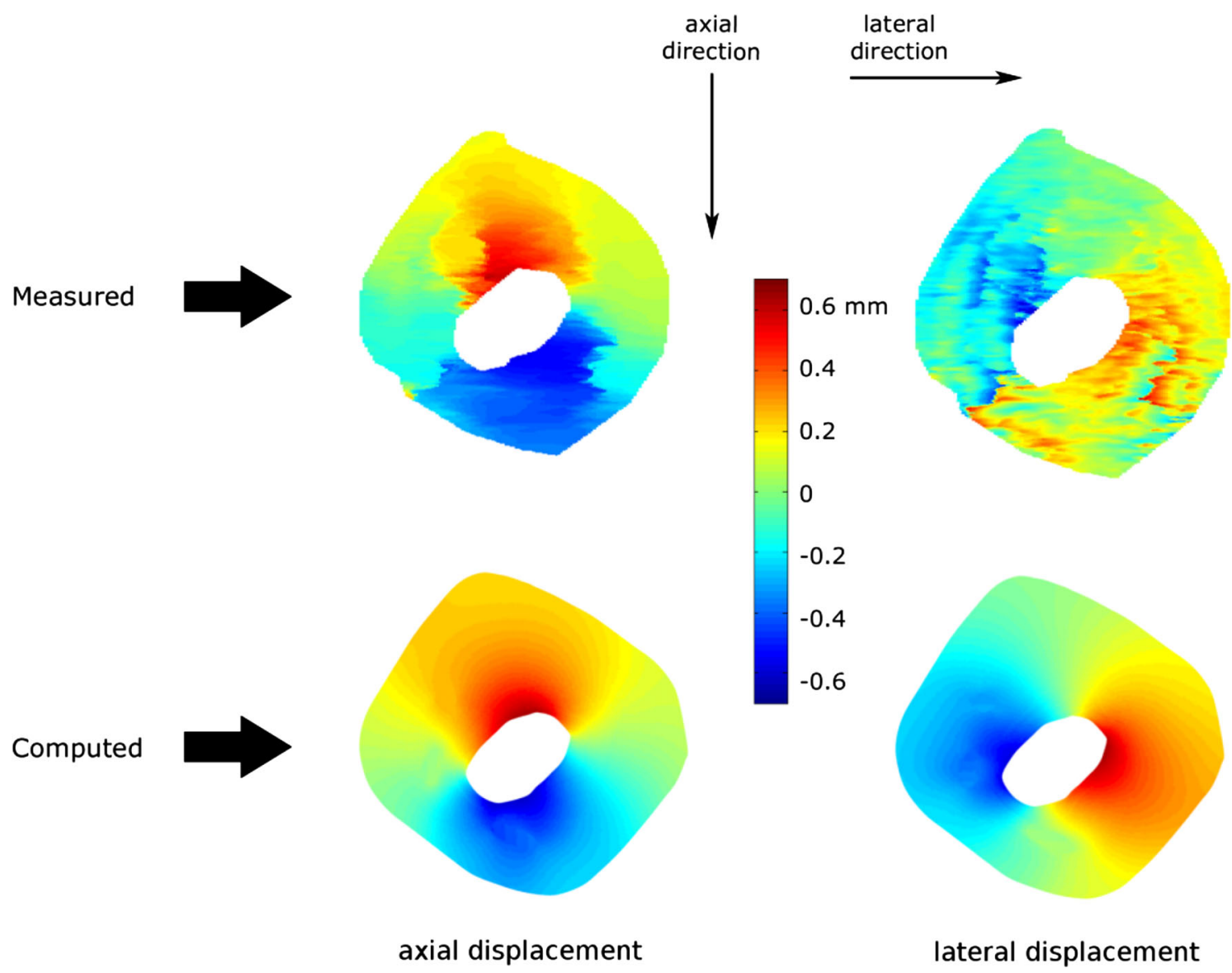

FIGURE 4. Measured (upper panel) and computed (lower panel) displacement maps in the axial direction (parallel to the ultrasound beam) in the left panel and lateral direction (perpendicular to the ultrasound beam) in the right panel for plaque 3 due to intraluminal pressure increase for pressure step 1 (from 10 to $80 \mathrm{mmHg}$ ).

pressure step 1, the displacement of point A was measured as $+0.20 \mathrm{~mm}$ whereas point B displaced by $+0.56 \mathrm{~mm}$. This implies that the tissue between point $\mathrm{A}$ and $\mathrm{B}$ was compressed by $0.36 \mathrm{~mm}$. Similarly, the tissue between the point $\mathrm{C}$ and $\mathrm{D}$ was compressed by $0.20 \mathrm{~mm}$. The computed displacements showed a very good agreement with the measured displacements. For this plaque and pressure step 1, the relative difference between the measured and computed displacement, $\Delta u_{\text {rel }}$, was $0.8 \%$. Not only the general deformation pattern, but also local deformations were captured with both the ultrasound measurements and FE simulations. The plateau in the measured displacements between the points $\mathrm{C}$ and $\mathrm{D}$ suggests that this region showed a relatively small compression. Inspection of the histology and ultrasound images confirmed the presence of calcium in this region (Fig. 2). For pressure steps 2 and 3, the measured displacements were lower than for pressure step 1 as the pressure increase for step 2 and 3 was lower. The tissue region between A and $\mathrm{B}$ was compressed by $0.023 \mathrm{~mm}$ during pressure step 2 and by $0.019 \mathrm{~mm}$ during pressure step 3 . The lower compression in pressure step 2 compared to step 3 indicates that the tissue stiffened as the pressure increased. $\Delta u_{\text {rel }}$ for pressure steps 2 and 3 were 0.9 and $0.8 \%$, respectively. For all plaques combined, the average [range] $\Delta u_{\text {rel }}$ for pressure step 1,2 and 3 were $1.1 \%[0.5-1.2 \%], 2.7 \%[0.9-6.4 \%]$, and $2.4 \%[0.8-$ $6.2 \%$, respectively. Out of 18 cases (three pressure steps for six plaques), 14 had $\Delta u_{\text {rel }}$ lower than $2 \%$ whereas the remaining four steps (step 2 and 3 for plaques 1 and 4 ) had $\Delta u_{\text {rel }}$ greater than $4.5 \%$.

Table 1 displays the estimated Young's modulus values for atherosclerotic intima and wall for the 6 models together with the mean values. Both plaque components showed increasing stiffness with increasing pressure. The mean Young's modulus ( \pm standard deviation) for intima was $24( \pm 17) \mathrm{kPa}$ for the pressure change from 10 to $80 \mathrm{mmHg}, 100( \pm 68) \mathrm{kPa}$ for the pressure change from 80 to $100 \mathrm{mmHg}$, and 190 $( \pm 187) \mathrm{kPa}$ for the pressure increase from 100 to $120 \mathrm{mmHg}$. On average, arterial wall was stiffer than intima for all pressure steps. For the wall, mean Young's modulus values increased from $142( \pm 108)$ to 


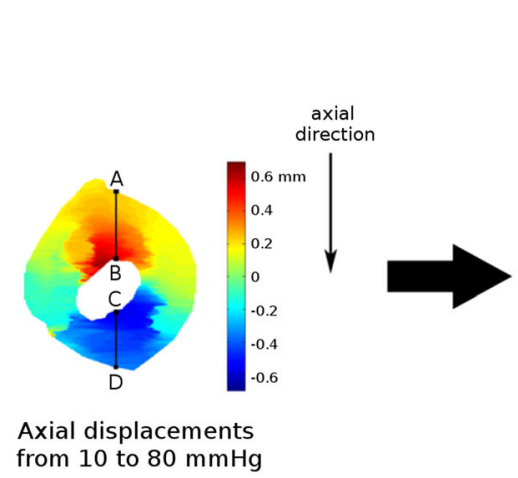

Axial displacements
from 10 to $80 \mathrm{mmHg}$

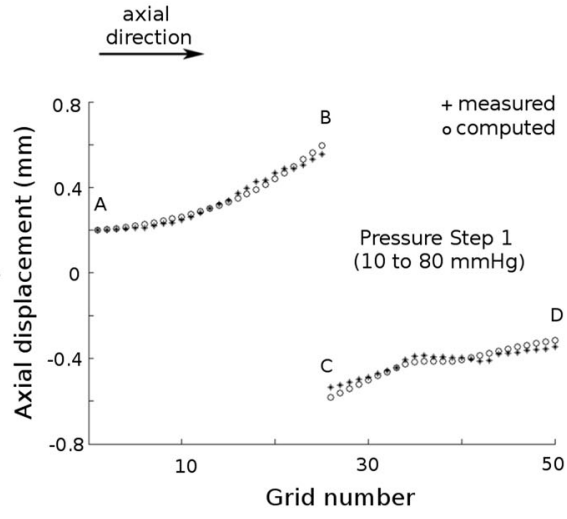

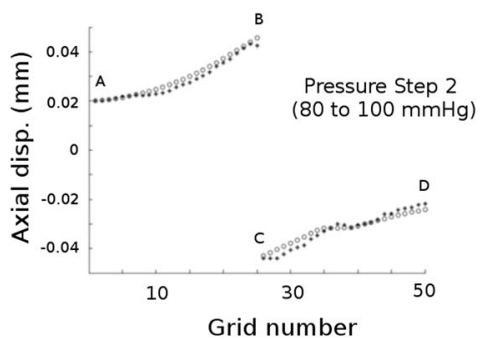

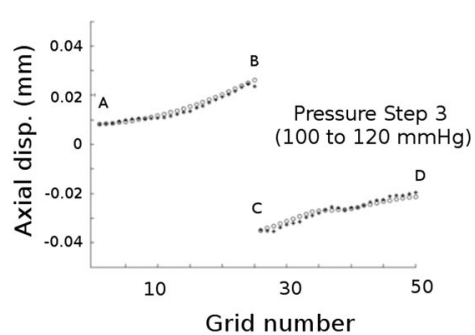

FIGURE 5. Measured (solid dots) and computed (circles) axial displacements in the midsection of plaque 3 due to intraluminal pressure increase during inflation. Please note the difference in the $y$-axis scaling for the three pressure steps.

$877( \pm 459) \mathrm{kPa}$, and then to $1015( \pm 439) \mathrm{kPa}$ for the same sequence of pressure steps. The estimated stiffness for the individual plaques showed a wide variation but consistent behavior. Two exceptions were observed: for plaque 1, the stiffness of the wall slightly decreased when going from pressure step 2 to step 3 . For plaque 4, the intima showed very low stiffness values, hitting the lower limit for pressure step 2 and 3 . It should be noted that these four cases are the ones that resulted in larger $\Delta \mathrm{u}_{\text {rel }}$ values $(>4.5 \%)$ compared to the other 14 cases $(<2 \%)$. If these 4 cases are excluded from the analysis, the mean Young's modulus ( \pm standard deviation) for intima was calculated 135 $( \pm 47) \mathrm{kPa}$ for the pressure step 2, and $149( \pm 39) \mathrm{kPa}$ for the pressure step 3, and for the atherosclerotic arterial wall, $678( \pm 332) \mathrm{kPa}$ for the pressure step 2, and $879( \pm 290) \mathrm{kPa}$ for the pressure step 3.

\section{DISCUSSION}

This study presented a new hybrid experimentalnumerical approach to determine local mechanical plaque properties. An RF-correlation technique was applied to high frequency ultrasound data to quantify plaque deformation at a high spatial resolution during inflation. Experiments with intact arteries enabled testing plaques with preserved structural integrity. By registering histology images to ultrasound images unprecedented details on complex plaque morphology were incorporated in the FE models. These models were used in an inverse FE method technique to estimate the material parameters of the arterial wall and intima of atherosclerotic plaques. This novel technique was utilized to characterize 6 porcine iliac atherosclerotic plaques from ex vivo inflation tests.

Accurate quantification of the local displacement distribution is essential for application of inverse methods to map the local properties of complex structures like atherosclerotic plaques. To measure the local displacement fields in the atherosclerotic plaques, a previously developed ultrasound RF cross correlation technique was applied to high-frequency ultrasound data for the first time. This technique was previously validated with vessel phantoms. ${ }^{17}$ An axial displacement estimation RMSE (root-mean-squared error) of $0.63 \mu \mathrm{m}$ was achieved in this validation study at an ultrasonic signal-to-noise level of $20 \mathrm{~dB}$ using a linear array transducer with a central frequency of 7.5 MHz. Although not verified, a smaller RMSE is anticipated with the high frequency ultrasound transducer used in the current study. This RF cross correlation technique combined with high frequency ultrasound resulted in displacement maps with an axial resolution of $15 \mu \mathrm{m}$, enabling us to image local heterogeneities over spatial scales that are relevant for atherosclerotic plaques. Not only are these high resolution measurements essential for the current application, they will also be essential if we want to study previously reported inhomogeneities within the intima. ${ }^{7}$

A high accuracy in matching the simulated displacement field to the measured data $\left(\Delta u_{\text {rel }}<2 \%\right)$ was achieved for 14 cases out 18 cases. This indicates that the underlying plaque model captures the experimental data very well for these cases. This observation has two implications. The first implication is that the registra- 


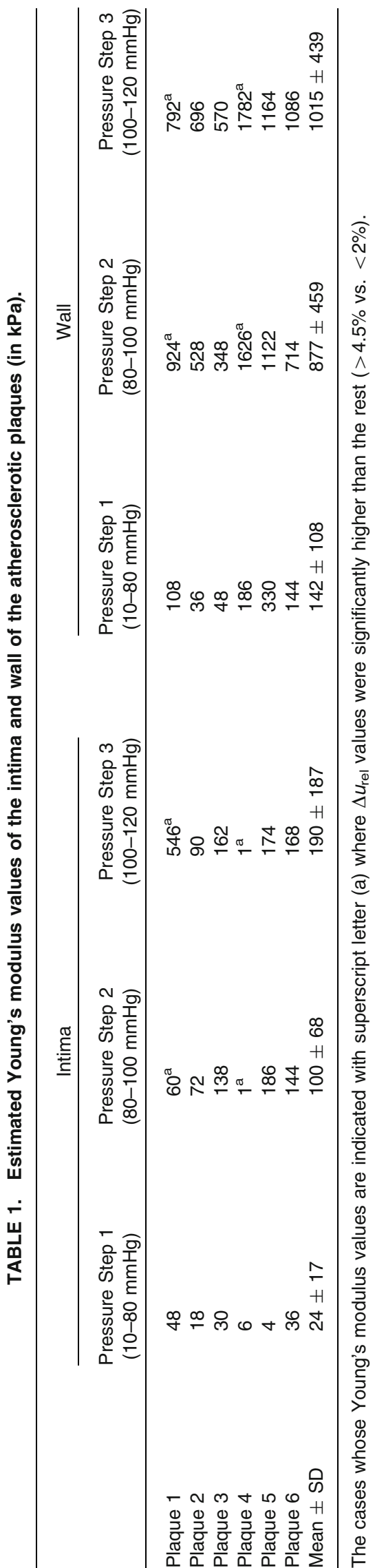

tion between the histology data and the ultrasound data, based on only the lumen and outer wall contour information, resulted in a good approximation of the underlying plaque geometry in the central region of the plaque. The second implication is that the neo-Hookean models used for the arterial wall and the intima captured the mechanical response in the central region of the plaque to the given intraluminal pressure very well. Employing a more nonlinear material model was possible, however, not preferred in this study as the aim was to develop a framework to estimate local stiffness values. To demonstrate the feasibility of this framework, a single parameter neo-Hookean material model for the diseased intima and the wall was employed. Fitting a multiparameter nonlinear material model to the strain data may result in overparameterization and therefore nonunique solutions. ${ }^{6,42,46}$ To evaluate nonlinear stiffening of the tissue, an incremental stiffness assessment was done by fitting the neoHookean model to strain data from three incremental pressure steps.

On average, both the intima and the wall exhibited the expected nonlinear behavior: ${ }^{1}$ the stiffness increased with increasing pressure. Furthermore, the average stiffness values for the wall were higher than the stiffness value for the intima component. This indicates that the arterial wall was the major load bearing structure in these atherosclerotic iliac plaques. The average wall stiffness values are similar to healthy porcine aorta wall properties obtained from inflation tests. ${ }^{22,30}$ The average intima stiffness values are in the lower range of human data in literature, ${ }^{1}$ and similar to the compressive stiffness values reported for aortic plaques $^{27}$ and tensile stiffness for human carotid intima tissue. ${ }^{10}$ The histology examination of the tested porcine plaques revealed that these plaques show pathological features of early stage human plaques, hence low stiffness results are not surprising. The average data however cannot conceal the considerable variation in the intima and wall properties. Even in plaques, harvested from the same vascular territory from animals of the same age that were exposed to the same diet, stiffness values span a wide range. This implies that for accurate modeling of the stress distribution in atherosclerotic plaques, plaque specific properties should be used.

The new hybrid experimental-numerical approach has the potential to be extended to in vivo use. To translate the technique to carotid plaque assessment, high resolution strain/displacement imaging and plaque composition data are required. Resolution of about $0.2 \times 0.2 \mathrm{~mm}^{2}$, which is not that far from the resolution used in this study, can be obtained if a clinical linear-array transducer with $9 \mathrm{MHz}$ center frequency is used to measure displacement field in 
carotid plaques. Obtaining high resolution plaque morphology data to replace histology is more challenging, but magnetic resonance imaging (MRI) can provide this information. In a recent study we demonstrated that a comparable approach using ultrasound based strain fields and plaque composition from MRI is fairly robust and insensitive to small segmentation errors. ${ }^{33}$ To determine coronary plaque properties, invasive imaging techniques have to be used as coronary arteries are too small and far from body surface. Intravascular ultrasound virtual histology (IVUS-VH) can be employed to determine the morphology and intravascular ultrasound palpography (IVUS Palpography) to measure the deformation. Combination of the two imaging modalities was utilized before ${ }^{40}$ and can provide the required data set for application of this framework in coronary arteries. Engagement of optical coherence tomography (OCT) can offer further improvement in imaging the luminal side of plaques, which would be very beneficial especially for characterization of thin cap fibro-atheromas. $^{13}$

Some limitations of the method presented in this study should be noted. First, only axial displacements were utilized in the minimization procedure as lateral displacement measurements by ultrasound were shown before to be much noisier than axial displacements. ${ }^{16,29,31}$ To obtain accurate lateral displacement from ultrasound recordings more advanced displacement estimation techniques such as spatial compounding technique are required, ${ }^{17,25}$ which have not been implemented yet for high frequency ultrasound systems such as the one used in our study. We used displacement measurements only from the mid-section of the plaque cross-sections since the most accurate ultrasound displacement measurements are collected in the central region of the ultrasound beam. To test how the stiffness results from the optimization procedure can be affected by the region size, we conducted a pilot study on plaque \#3. Using axial displacements not only from the mid-section but the entire plaque cross-section in the optimization did not change stiffness results, only the relative error increased from 0.8 to $4.8 \%$ as noisier data from the side regions were included. Same stiffness results when full plaque cross-section incorporated can be explained by the relatively simple morphology of the plaques tested. The qualitative histological investigation showed that the tested porcine iliac plaques were fairly homogeneous. Although this observation corroborates modeling the atherosclerotic intima homogenously for the tested plaques, in vivo application of the proposed method to advanced human plaques would require $2 \mathrm{D}$ displacement field of the entire plaque cross-section as they are morphologically and possibly also mechanically more inhomogeneous. In the future we plan to study the added value of full $2 \mathrm{D}$ displacement measurements to estimating material properties of plaque components and if it is required to model the inhomogeneity of advanced atherosclerotic plaques. Secondly, the software elastix, which was used to register histology images to ultrasound images, employs a purely image based algorithm and does not incorporate any structural, physical information to compute the transformation matrix for the registration. This might lead to some local errors in the registration. However, in our study successful registration for 14 out of 18 plaques was confirmed by the low relative difference between the measured and computed displacement fields in the central region. A representative example of a case with low relative difference can be seen in Fig. 5. Thirdly, possible anisotropic material behavior of intima and wall components was neglected in the study. Yet the isotropic Neo-Hookean model, used in FE simulations, resulted in low relative differences between the measured and computed displacement values. More advanced material models can demonstrate the material behavior of atherosclerotic plaque components better and can be employed in the FE models if needed. Similarly, the method presented in this paper allows including more components in the FE models if desired. For instance, although media and adventitia layers are structurally and mechanically two distinct layers, they are fused into one effective arterial wall component as the primary focus of the study was the demonstration of a new method for mechanical characterization of atherosclerotic plaque components, especially atherosclerotic intima. Yet, the proposed technique allows including them separately in the FE models with the cost of more intensive computations. Plane strain assumption is used in the study. The vessel segments were prestretched longitudinally before inflation and kept at constant length during inflation. No appreciable axial displacements were observed visually during the experiments and qualitative assessment of the B-mode images at different pressures did not show any change in the overall appearance of the imaged cross-section. Good correlation between the RF data collected at different pressure steps also confirms this. Therefore, plane strain assumption seems to be justified for this study. Another limitation of the study is that the residual stresses are neglected. Some interesting approaches were proposed earlier on how to investigate residual strains/stresses in atherosclerotic plaques, ${ }^{34,35}$ yet the advancement on this issue is very limited as the atherosclerotic plaque structure is very complex, heterogeneous and nonuniform. Therefore, residual strains/stresses were not incorporated in the current study. The test were performed at room temperature as Schaar et al. demon- 
strated that the difference between the strain data collected from in vitro inflation experiments of atherosclerotic coronary arteries at room temperature and body temperature were not statistically different. ${ }^{38}$ Inflation procedure leaves the arterial segment intact, which is one of the main advantages of this method. A disadvantage is that, if the plaque is relatively small, the intima will not have a major impact on the displacement field, and as a consequence, the outcome of the minimization procedure will be relatively insensitive to the stiffness of the intima.

In conclusion, we developed a new hybrid experimental-numerical technique to characterize local mechanical properties of atherosclerotic plaques. The combination of geometrical data from histology and high resolution displacement data from ultrasound was used to quantify the properties of intima and the wall atherosclerotic iliac arteries from six pigs. Both components exhibited nonlinear behavior and on average, the wall was stiffer than the intima. A large variation in the properties of the intima was observed, warranting further research into the relationship between stiffness and compositions of the intima.

\section{OPEN ACCESS}

This article is distributed under the terms of the Creative Commons Attribution 4.0 International License (http://creativecommons.org/licenses/by/4.0/), which permits unrestricted use, distribution, and reproduction in any medium, provided you give appropriate credit to the original author(s) and the source, provide a link to the Creative Commons license, and indicate if changes were made.

\section{REFERENCES}

\footnotetext{
${ }^{1}$ Akyildiz, A. C., L. Speelman, and F. J. Gijsen. Mechanical properties of human atherosclerotic intima tissue. J. Biomech. 47:773-783, 2014.

${ }^{2}$ Akyildiz, A. C., L. Speelman, H. van Brummelen, M. A. Gutierrez, R. Virmani, A. van der Lugt, et al. Effects of intima stiffness and plaque morphology on peak cap stress. Biomed. Eng. Online. 10:25, 2011.

${ }^{3}$ Baldewsing, R. A., M. G. Danilouchkine, F. Mastik, J. A. Schaar, P. W. Serruys, and A. F. van der Steen. An inverse method for imaging the local elasticity of atherosclerotic coronary plaques. IEEE Trans. Inf. Technol. Biomed. 12:277-289, 2008.

${ }^{4}$ Beattie, D., C. Xu, R. Vito, S. Glagov, and M. C. Whang. Mechanical analysis of heterogeneous, atherosclerotic human aorta. J. Biomech. Eng. 120:602-607, 1998.
}

${ }^{5}$ Bouvier, A., F. Deleaval, M. M. Doyley, S. K. Yazdani, G. Finet, S. Le Floc'h, et al. A direct vulnerable atherosclerotic plaque elasticity reconstruction method based on an original material-finite element formulation: theoretical framework. Phys. Med. Biol. 58:8457-8476, 2013.

${ }^{6}$ Carniel, E. L., C. G. Fontanella, L. Polese, S. Merigliano, and A. N. Natali. Computational tools for the analysis of mechanical functionality of gastrointestinal structures. Technol. Health Care 21:271-283, 2013.

${ }^{7}$ Chai, C. K., A. C. Akyildiz, L. Speelman, F. J. Gijsen, C. W. Oomens, M. R. van Sambeek, et al. Local axial compressive mechanical properties of human carotid atherosclerotic plaques-characterisation by indentation test and inverse finite element analysis. J. Biomech. 46:17591766, 2013.

${ }^{8}$ Chandran, K. B., J. H. Mun, K. K. Choi, J. S. Chen, A. Hamilton, A. Nagaraj, et al. A method for in vivo analysis for regional arterial wall material property alterations with atherosclerosis: preliminary results. Med. Eng. Phys. 25:289-298, 2003.

${ }^{9}$ Cheng, G. C., H. M. Loree, R. D. Kamm, M. C. Fishbein, and R. T. Lee. Distribution of circumferential stress in ruptured and stable atherosclerotic lesions. A structural analysis with histopathological correlation. Circulation 87:1179-1187, 1993.

${ }^{10}$ Cunnane, E. M., J. J. Mulvihill, H. E. Barrett, D. A. Healy, E. G. Kavanagh, S. R. Walsh, et al. Mechanical, biological and structural characterization of human atherosclerotic femoral plaque tissue. Acta Biomater. 11:295-303, 2014.

${ }^{11}$ Falk, E. Pathogenesis of atherosclerosis. J. Am. Coll. Cardiol. 47:C7-C12, 2006.

${ }^{12}$ Franquet, A., S. Avril, R. Le Riche, and P. Badel. Identification of heterogeneous elastic properties in stenosed arteries: a numerical plane strain study. Comput. Methods Biomech. Biomed. Eng. 15:49-58, 2011.

${ }^{13}$ Fujii, K., D. Kawasaki, M. Masutani, T. Okumura, T. Akagami, T. Sakoda, et al. OCT assessment of thin-cap fibroatheroma distribution in native coronary arteries. JACC Cardiovasc. Imaging. 3:168-175, 2010.

${ }^{14}$ Fung, Y. C., and S. Q. Liu. Determination of the mechanical properties of the different layers of blood vessels in vivo. Proc. Natl. Acad. Sci. USA. 92:2169-2173, 1995.

${ }^{15}$ Hamilton, A. J., H. Kim, A. Nagaraj, J. H. Mun, L. L. Yan, S. I. Roth, et al. Regional material property alterations in porcine femoral arteries with atheroma development. J. Biomech. 38:2354-2364, 2005.

${ }^{16}$ Hansen, H. H., R. G. Lopata, and C. L. de Korte. Noninvasive carotid strain imaging using angular compounding at large beam steered angles: validation in vessel phantoms. IEEE Trans. Med. Imaging 28:872-880, 2009.

${ }^{17}$ Hansen, H. H., R. G. Lopata, T. Idzenga, and C. L. de Korte. Full 2D displacement vector and strain tensor estimation for superficial tissue using beam-steered ultrasound imaging. Phys. Med. Biol. 55:3201-3218, 2010.

${ }^{18}$ Holzapfel, G. A., J. J. Mulvihill, E. M. Cunnane, and M. T. Walsh. Computational approaches for analyzing the mechanics of atherosclerotic plaques: a review. J. Biomech. 47:859-869, 2014.

${ }^{19}$ Humphrey, J. D. Cardiovascular solid mechanics: cells, tissues and organs. New York: Springer, 2002.

${ }^{20}$ Karimi, R., T. Zhu, B. E. Bouma, and M. R. Mofrad. Estimation of nonlinear mechanical properties of vascular tissues via elastography. Cardiovasc. Eng. 8:191-202, 2008. 
${ }^{21}$ Khalil, A. S., R. C. Chan, A. H. Chau, B. E. Bouma, and M. R. Mofrad. Tissue elasticity estimation with optical coherence elastography: toward mechanical characterization of in vivo soft tissue. Ann. Biomed. Eng. 33:1631-1639, 2005.

${ }^{22} \mathrm{Kim}$, J., and S. Baek. Circumferential variations of mechanical behavior of the porcine thoracic aorta during the inflation test. J. Biomech. 44:1941-1947, 2011.

${ }^{23}$ Klein, S., M. Staring, K. Murphy, M. A. Viergever, and J. P. Pluim. Elastix: a toolbox for intensity-based medical image registration. IEEE Trans. Med. Imaging 29:196-205, 2010.

${ }^{24}$ Koopmans, S. J., R. Dekker, M. T. Ackermans, H. P. Sauerwein, M. J. Serlie, H. M. van Beusekom, et al. Dietary saturated fat/cholesterol, but not unsaturated fat or starch, induces C-reactive protein associated early atherosclerosis and ectopic fat deposition in diabetic pigs. Cardiovasc. Diabetol. 10:64, 2011.

${ }^{25}$ Korukonda, S., R. Nayak, N. Carson, G. Schifitto, V. Dogra, and M. M. Doyley. Noninvasive vascular elastography using plane-wave and sparse-array imaging. IEEE Trans. Ultrason. Ferroelectr. Freq. Control 60:332-342, 2013.

${ }^{26}$ Le Floc'h, S., J. Ohayon, P. Tracqui, G. Finet, A. M. Gharib, R. L. Maurice, et al. Vulnerable atherosclerotic plaque elasticity reconstruction based on a segmentationdriven optimization procedure using strain measurements: theoretical framework. IEEE Trans. Med. Imaging 28:1126-1137, 2009.

${ }^{27}$ Lee, R. T., S. G. Richardson, H. M. Loree, A. J. Grodzinsky, S. A. Gharib, F. J. Schoen, et al. Prediction of mechanical properties of human atherosclerotic tissue by high-frequency intravascular ultrasound imaging. An in vitro study. Arterioscler. Thromb. 12:1-5, 1992.

${ }^{28}$ Liu, H., G. Canton, C. Yuan, C. Yang, K. Billiar, Z. Teng, et al. Using in vivo Cine and $3 \mathrm{D}$ multi-contrast MRI to determine human atherosclerotic carotid artery material properties and circumferential shrinkage rate and their impact on stress/strain predictions. J. Biomech. Eng. 134:011008, 2012.

${ }^{29}$ Lopata, R. G., M. M. Nillesen, H. H. Hansen, I. H. Gerrits, J. M. Thijssen, and C. L. de Korte. Performance evaluation of methods for two-dimensional displacement and strain estimation using ultrasound radio frequency data. Ultrasound Med. Biol. 35:796-812, 2009.

${ }^{30}$ Lopata, R. G., M. F. Peters, J. Nijs, C. W. Oomens, M. C. Rutten, and F. N. van de Vosse. Vascular elastography: a validation study. Ultrasound Med. Biol. 40:1882-1895, 2014.

${ }^{31}$ Luo, J., and E. E. Konofagou. Effects of various parameters on lateral displacement estimation in ultrasound elastography. Ultrasound Med. Biol. 35:1352-1366, 2009.

${ }^{32}$ Nagaraj, A., H. Kim, A. J. Hamilton, J. H. Mun, B. Smulevitz, B. J. Kane, et al. Porcine carotid arterial material property alterations with induced atheroma: an in vivo study. Med. Eng. Phys. 27:147-156, 2005.
${ }^{33}$ Nieuwstadt, H. A., S. Fekkes, H. H. G. Hansen, C. De Korte, A. van der Lugt, J. J. Wentzel, et al. Carotid plaque elasticity estimation using ultrasound elastography, MRI, and inverse FEA - a numerical feasibility study. Med. Eng. Phys., 2015, in press.

${ }^{34}$ Ohayon, J., O. Dubreuil, P. Tracqui, S. Le Floc'h, G. Rioufol, L. Chalabreysse, et al. Influence of residual stress/ strain on the biomechanical stability of vulnerable coronary plaques: potential impact for evaluating the risk of plaque rupture. Am. J. Physiol. Heart Circ. Physiol. 293:H1987-H1996, 2007.

${ }^{35}$ Ohayon, J., N. Mesnier, A. Broisat, J. Toczek, L. Riou, and $\mathrm{P}$. Tracqui. Elucidating atherosclerotic vulnerable plaque rupture by modeling cross substitution of $\mathrm{ApoE}^{-/-}$ mouse and human plaque components stiffnesses. Biomech. Model. Mechanobiol. 11:801-813, 2012.

${ }^{36}$ Pandit, A., X. Lu, C. Wang, and G. S. Kassab. Biaxial elastic material properties of porcine coronary media and adventitia. Am. J. Physiol. Heart Circ. Physiol. 288:H2581H2587, 2005.

${ }^{37}$ Richardson, P. D., M. J. Davies, and G. V. Born. Influence of plaque configuration and stress distribution on fissuring of coronary atherosclerotic plaques. Lancet 2:941-944, 1989.

${ }^{38}$ Schaar, J. A., C. L. de Korte, F. Mastik, and A. F. van der Steen. Effect of temperature increase and freezing on intravascular elastography. Ultrasonics 40:879-881, 2002.

${ }^{39}$ Schaar, J. A., J. E. Muller, E. Falk, R. Virmani, V. Fuster, P. W. Serruys, et al. Terminology for high-risk and vulnerable coronary artery plaques. Report of a meeting on the vulnerable plaque, June 17 and 18, 2003, Santorini. Greece. Eur. Heart J. 25:1077-1082, 2004.

${ }^{40}$ Serruys, P. W., H. M. Garcia-Garcia, P. Buszman, P. Erne, S. Verheye, M. Aschermann, et al. Effects of the direct lipoprotein-associated phospholipase $\mathrm{A}(2)$ inhibitor darapladib on human coronary atherosclerotic plaque. Circulation 118:1172-1182, 2008.

${ }^{41}$ Speelman, L., A. C. Akyildiz, B. den Adel, J. J. Wentzel, A. F. van der Steen, R. Virmani, et al. Initial stress in biomechanical models of atherosclerotic plaques. J. Biomech. 44:2376-2382, 2011.

${ }^{42}$ Stalhand, J., A. Klarbring, and M. Karlsson. Towards in vivo aorta material identification and stress estimation. Biomech. Model. Mechanobiol. 2:169-186, 2004.

${ }^{43}$ Tian, L., and N. C. Chesler. In vivo and in vitro measurements of pulmonary arterial stiffness: a brief review. Pulm. Circ. 2:505-517, 2013.

${ }^{44}$ Virmani, R., A. V. Finn, and F. D. Kolodgie. Carotid plaque stabilization and progression after stroke or TIA. Arterioscler. Thromb. Vasc. Biol. 29:3-6, 2009.

${ }^{45}$ Walsh, M. T., E. M. Cunnane, J. J. Mulvihill, A. C. Akyildiz, F. J. Gijsen, and G. A. Holzapfel. Uniaxial tensile testing approaches for characterisation of atherosclerotic plaques. J. Biomech. 47:793-804, 2014.

${ }^{46}$ Yin, F. C., P. H. Chew, and S. L. Zeger. An approach to quantification of biaxial tissue stress-strain data. J. Biomech. 19:27-37, 1986. 